\title{
Efeitos de períodos sem a ocorrência de chuva na eficácia do flumioxazin aplicado no solo e na palha de cana-de-açúcar ${ }^{1}$
}

\author{
Effects of different periods without rains occurrence in the efficacy of flumioxazin \\ applied in soil and in the sugarcane mulch
}

\section{Caio Antonio Carbonari ${ }^{2}$; Giovanna Larissa Gimenes Cotrick Gomes ${ }^{3}$; Edivaldo Domingues Velini ${ }^{4}$.}

\begin{abstract}
Resumo - O presente trabalho teve por objetivo avaliar a eficácia do flumioxazin aplicado sobre o solo e em associação com a palha de cana-de-açúcar, seguido de diferentes períodos sem ocorrência de chuvas. O experimento foi conduzido em vasos em casa-de-vegetação, no município de Botucatu/SP. Os vasos foram preenchidos com solo e as plantas daninhas Brachiaria decumbens, Digitaria horizontalis, Ipomoea nil, Ipomoea grandifolia, Bidens pilosa e Sida rhombifolia, foram semeadas e em seguida cobertos ou não com palha de cana-de-açúcar, dependendo do tratamento utilizado. O delineamento experimental utilizado foi inteiramente casualizado com quatro repetições. Os tratamentos foram dispostos em esquema fatorial $4 \times 2$, sendo os fatores, quatro períodos sem ocorrência de chuvas, 1, 15, 30 e 60 dias e dois posicionamentos do produto, sobre o solo sem palha e sobre a palha de cana-de-açúcar. Foram realizadas avaliações de controle aos 14, 28 e 42 dias após a ocorrência da chuva. De maneira geral, pode-se observar que a aplicação do flumioxazin sobre o solo ou sobre a palha de cana-deaçúcar promoveu bons níveis de controle das plantas daninhas. Foram observadas reduções nos níveis de controle para algumas espécies de plantas daninhas, quando o produto foi exposto ao período de 60 dias sem ocorrência de chuvas.
\end{abstract}

Palavras-chaves: controle químico, cobertura morta, plantas daninhas.

\footnotetext{
Abstract - The present work aimed to evaluate the efficacy of flumioxazin applied over the soil or in association with sugarcane mulch, followed by different periods without the rain occurrence. The experiment was conducted in vases under greenhouse conditions in Botucatu County, São Paulo, Brazil. Vases were filled with soil and Brachiaria decumbens, Digitaria horizontalis, Ipomoea nil, Ipomoea grandifolia, Bidens pilosa and Sida rhombifolia weeds were sown and then covered or not with sugarcane mulch, depending on the treatment used. The experimental design used was entirely randomized with four replications. Treatments were

${ }^{1}$ Recebido para publicação em 27/12/2010 e na forma revisada em 20/02/2011.

${ }^{2}$ Engenheiro Agrônomo Dr., FCA/UNESP, Depto. de Produção Vegetal, Faculdade de Ciências Agronômicas -

FCA/UNESP, 18603-970, Botucatu-SP, carbonari@fca.unesp.br.

3 Mestranda em Agronomia, FCA/UNESP, Depto. de Produção Vegetal, Faculdade de Ciências Agronômicas FCA/UNESP, 18603-970, Botucatu-SP, giovanna.gomes@ fca.unesp.br.

4 Professor Livre-Doente, FCA/UNESP, Depto. de Produção Vegetal, Faculdade de Ciências Agronômicas FCA/UNESP, 18603-970, Botucatu-SP, velini@fca.unesp.br.
} 
Carbonari et al.

displaced in $4 \times 2$ factorial scheme, with factors representing four periods without rain occurrence, 1, 15, 30 and 60 days and two herbicides positioning, over the soil without sugarcane mulch and over sugarcane mulch. Control evaluations were carried out at 14, 28 and 42 days after rain occurrence. In general mode, it was possible to observe that flumioxazin application over the soil or over sugarcane mulch promoted control satisfactory levels of weeds. Reductions on control levels of some weed species were observed when the herbicide was exposed to a period of 60 days without rain occurrence.

Key-words: chemical control, mulch, weeds.

\section{Introdução}

O controle químico de plantas daninhas em áreas de cana-de-açúcar é uma prática bastante difundida, sendo utilizados, em geral, herbicidas de ação em pré e pós-emergência inicial, recomendados para controle de gramíneas, folhas largas e plantas perenes de difícil controle. Segundo Miller et al. (1995), a utilização de herbicidas pré-emergentes com efeito residual prolongado é um dos fatores que determinam a eficácia no controle de plantas daninhas durante $\mathrm{o}$ período crítico de competição. No entanto, com o aumento de áreas de cana-de-açúcar colhidas mecanicamente e com depósito dos restos culturais sobre o solo, sistema denominado de cana crua, a dinâmica e interação destes herbicidas com a palha de cana-de-açúcar promovem alterações no comportamento e eficácia dos produtos. Maciel \& Velini (2005) e Simoni et al. (2006), relatam que a manutenção da palha sobre a superfície do solo pode, simultaneamente, reduzir o potencial de infestação das plantas daninhas, bem como dificultar o desempenho dos herbicidas, uma vez que a água da chuva se torna a principal responsável pelo transporte do herbicida até a superfície do solo.

O comportamento de herbicidas residuais aplicados sobre a cobertura morta não depende apenas das características específicas do produto, mas também da quantidade e origem dessa cobertura, volume de água e época da primeira irrigação ocorrida após a aplicação do produto, assim como das irrigações subseqüentes, condições climáticas prevalecentes durante e após a aplicação, entre outros (Rodrigues, 1993). Segundo Watts \& Hall (1996), a eficácia na utilização de um herbicida aplicado sobre a cobertura morta depende de vários fatores, principalmente fatores ambientais. Dentre os fatores ambientais, o que afeta decisivamente a capacidade de um herbicida aplicado em préemergência atuar no controle de plantas daninhas, atingindo o solo é a precipitação.

Lamoreaux et al. (1993) ressaltam que o transporte de herbicidas da palha para o solo é dependente da capacidade da palha de cobrir o solo e reter estes herbicidas, das características físico-químicas do herbicida, bem como do período em que a área permanece sem chuva após a aplicação. Para diversos herbicidas verifica-se que a permanência sobre a palha por longos períodos sem chuvas, implica em reduções nas quantidades do herbicida carregado ao solo pela primeira chuva e, consequentemente, na eficácia do herbicida no controle das plantas daninhas (Godoy et al., 2007; Cavenaghi et al., 2007; Tofoli, 2009; Carbonari et al., 2010).

$\mathrm{O}$ flumioxazin [N-(7-fluoro-3,4dihydro-3-oxo-4-prop-2-ynyl-2H-1,4-

benzoxazin-6-yl) cyclohex-1-ene-1,2dicarboxamide] é um herbicida registrado para uso em condições de pré-emergência, no controle de plantas daninhas de folhas largas e monocotiledôneas na cultura da cana-de- 
Carbonari et al.

açúcar. O flumioxazin é um produto não-iônico que apresenta baixa solubilidade em água $(1,79$ $\mathrm{mg} \mathrm{L}^{-1}$ a $25^{\circ} \mathrm{C}$ ) (Ferrell et al., 2005) e pressão de vapor $\left(2,41 \times 10^{-6} \mathrm{mmHg}\right.$ a $\left.22^{\circ} \mathrm{C}\right)$, o que sugere que ele tenha baixo potencial de volatilização (Rodrigues \& Almeida, 2005). No solo apresenta meia-vida de 21,9 dias (Rodrigues e Almeida, 2005), e sua degradação ocorre por hidrólise e atividade de microorganismos (Alister et al., 2008). Estas características fazem deste herbicida um produto com baixo risco de lixiviação (Ferrell et al., 2005; Alister et al., 2008).

O objetivo deste estudo foi avaliar os efeitos de diferentes períodos de permanência do flumioxazin na palha de cana-de-açúcar e no solo antes da ocorrência de chuvas, na eficácia de controle de plantas daninhas.

\section{Material e métodos}

Foi conduzido um experimento em casa-de-vegetação, no Núcleo de Pesquisas Avançadas em Matologia (NUPAM), pertencente à Faculdade de Ciências Agronômicas (FCA/UNESP) no município de Botucatu/SP. Vasos com dimensões de 15 x 15 $\mathrm{cm}\left(0,0225 \mathrm{~m}^{2}\right)$ e capacidade para 2 litros de solo foram utilizados como unidades expeerimentais, juntamente como o substrato, um Latossolo Vermelho Escuro (LVd), de textura média, com as seguintes características químicas e físicas: $\mathrm{pH}\left(\mathrm{CaCl}_{2}\right) 4,3$; M.O. $19 \mathrm{~g}$ $\mathrm{dm}^{-3}$; SB e CTC de 14,6 e 73, respectivamente, e $76 \%$ de areia, $20 \%$ de argila e $4 \%$ de silte. Realizou-se uma adubação de correção do solo para que as condições de fertilidade fossem melhoradas e proporcionasse uma melhor manutenção e desenvolvimento das plantas daninhas.

Foram semeadas as espécies de plantas daninhas Brachiaria decumbens, Digitaria horizontalis, Ipomoea nil, Ipomoea grandifolia, Bidens pilosa e Sida rhombifolia, adicionandose quantidades de sementes para que se obtivessem 25 plantas de cada espécie por vaso.

A quantidade de palha de cana-deaçúcar sobre o solo, utilizada para cada vaso, foi calculada para representar cinco toneladas de massa seca por hectare. Essa quantidade foi selecionada em função das informações disponíveis na literatura e de estudos anteriormente realizados, indicando ser essa quantidade uma das condições mais críticas em termos de manejo de plantas daninhas em cana crua, uma vez que é insuficiente para impedir o desenvolvimento das plantas daninhas, mas o bastante para reter quase que completamente a quantidade de calda aplicada (Velini \& Negrisoli, 2000).

A aplicação do herbicida e a simulação de chuva foram realizadas através de um equipamento instalado em ambiente fechado. $\mathrm{O}$ herbicida flumioxazin $\left(\right.$ Flumyzin $^{\circledR}$ ) foi aplicado na dose de $150 \mathrm{~g}$ i.a. ha ${ }^{-1}$, através de um pulverizador estacionário equipado com barra contendo quatro pontas XR11002, e sendo operado em pressão constante de 1,5 bar, pressurizado por ar comprimido, constituindo um consumo de calda de $200 \mathrm{~L} \mathrm{ha}^{-1}$.

Os tratamentos foram constituídos em um esquema fatorial $4 \times 2$, sendo quatro os períodos de aplicação antes da ocorrência de chuvas $(1,15,30$ e 60 dias) e dois os posicionamentos da aplicação (sobre o solo e sobre a palha de cana-de-açúcar), além de testemunhas sem aplicação do produto, na presença e ausência de palha de cana-deaçúcar. Após cada um dos períodos a quantidade de chuva simulada em cada tratamento foi de $20 \mathrm{~mm}$, e os vasos passaram a receber irrigação subsuperficial diariamente. Os tratamentos foram dispostos em delineamento inteiramente casualizado, com quatro repetições.

As avaliações de controle foram realizadas aos 14, 28 e 42 dias após a ocorrência da primeira chuva (DAC), através 
Carbonari et al.

de notas visuais, segundo escala percentual de notas, onde " $0 \%$ "correspondeu a nenhum controle e " $100 \%$ " ao controle total das plantas daninhas.

Os resultados obtidos foram submetidos à análise de variância pelo teste " $F$ " a $5 \%$ de probabilidade e as suas médias comparadas pelo teste Tukey, a 5\% de probabilidade.

\section{Resultados e discussão}

$\mathrm{Na}$ Tabela 1, estão apresentados os resultados de controle de $B$. decumbens e $D$. horizontalis pelo flumioxazin aplicado na

Tabela 1. Controle de B. decumbens e Digitaria horizontalis pelo herbicida flumioxazin em diferentes períodos de permanência na palha de cana-de-açúcar antes da ocorrência de chuvas. Botucatu/SP - 2009/10.

\begin{tabular}{|c|c|c|c|c|c|c|}
\hline \multirow[b]{2}{*}{ Períodos (dias) } & \multicolumn{2}{|c|}{$14 \mathrm{DAC}$} & \multicolumn{2}{|c|}{$28 \mathrm{DAC}$} & \multicolumn{2}{|c|}{$42 \mathrm{DAC}$} \\
\hline & $\begin{array}{l}\text { com } \\
\text { palha }\end{array}$ & $\begin{array}{l}\text { sem } \\
\text { palha }\end{array}$ & $\begin{array}{l}\text { com } \\
\text { palha }\end{array}$ & $\begin{array}{l}\text { sem } \\
\text { palha }\end{array}$ & $\begin{array}{l}\text { com } \\
\text { palha }\end{array}$ & $\begin{array}{l}\text { sem } \\
\text { palha }\end{array}$ \\
\hline & \multicolumn{6}{|c|}{ B. decumbens } \\
\hline 1 & $99,0 \mathrm{Aa}$ & $100,0 \mathrm{Aa}$ & $97,3 \mathrm{Aa}$ & $100,0 \mathrm{Aa}$ & $98,3 \mathrm{Aa}$ & $100,0 \mathrm{Aa}$ \\
\hline 15 & $98,3 \mathrm{Aa}$ & $100,0 \mathrm{Aa}$ & $98,0 \mathrm{Aa}$ & $100,0 \mathrm{Aa}$ & $98,0 \mathrm{Aa}$ & $100,0 \mathrm{Aa}$ \\
\hline 30 & $95,0 \mathrm{Ab}$ & 98,5 Aa & 98,3 Aa & $100,0 \mathrm{Aa}$ & $96,3 \mathrm{Aa}$ & $100,0 \mathrm{Aa}$ \\
\hline 60 & $96,3 \mathrm{Aa}$ & $97,5 \mathrm{Aa}$ & $90,0 \mathrm{Bb}$ & $100,0 \mathrm{Aa}$ & $88,8 \mathrm{Bb}$ & $100,0 \mathrm{Aa}$ \\
\hline $\mathrm{F}$ (posicion.) & & & & & & \\
\hline $\mathrm{F}$ (períodos) & & & & & & \\
\hline $\mathrm{F}$ (palha $\mathrm{x}$ per.) & & & & & & \\
\hline C.V. (\%) & & & & & & \\
\hline D.M.S.(posicion.) & & & & & & \\
\hline \multirow[t]{2}{*}{ D.M.S. (período) } & & & & & & \\
\hline & \multicolumn{6}{|c|}{ D. horizontalis } \\
\hline 1 & $99,0 \mathrm{Aa}$ & $100,0 \mathrm{Aa}$ & $100,0 \mathrm{Aa}$ & $100,0 \mathrm{Aa}$ & $\overline{100,0 \mathrm{Aa}}$ & $100,0 \mathrm{Aa}$ \\
\hline 15 & $99,5 \mathrm{Aa}$ & $100,0 \mathrm{Aa}$ & $99,3 \mathrm{Aa}$ & $100,0 \mathrm{Aa}$ & $98,0 \mathrm{ABa}$ & $100,0 \mathrm{Aa}$ \\
\hline 30 & $98,8 \mathrm{Aa}$ & $100,0 \mathrm{Aa}$ & $99,3 \mathrm{Aa}$ & $100,0 \mathrm{Aa}$ & $99,0 \mathrm{ABa}$ & $100,0 \mathrm{Aa}$ \\
\hline 60 & $98,3 \mathrm{Ab}$ & $100,0 \mathrm{Aa}$ & $98,3 \mathrm{Ab}$ & $100,0 \mathrm{Aa}$ & $96,3 \mathrm{Bb}$ & $100,0 \mathrm{Aa}$ \\
\hline $\mathrm{F}$ (posicion.) & \multicolumn{2}{|c|}{$7,97 * *$} & \multicolumn{2}{|c|}{$5,70^{*}$} & \multicolumn{2}{|c|}{$7.52 *$} \\
\hline $\mathrm{F}$ (períodos) & \multirow{2}{*}{\multicolumn{2}{|c|}{$\begin{array}{l}0,42^{\mathrm{ns}} \\
042^{\mathrm{ns}}\end{array}$}} & \multirow{2}{*}{\multicolumn{2}{|c|}{$1,11^{\mathrm{ns}}$}} & \multicolumn{2}{|c|}{$0,19^{\text {ns }}$} \\
\hline $\mathrm{F}$ (palha $\mathrm{x}$ per.) & & & & & \multirow{2}{*}{\multicolumn{2}{|c|}{$0,19^{-10}$}} \\
\hline C.V. $(\%)$ & \multicolumn{2}{|c|}{1,13} & \multicolumn{2}{|c|}{0,97} & & \\
\hline D.M.S.(posicion.) & \multicolumn{2}{|c|}{1,65} & \multicolumn{2}{|c|}{1,40} & \multicolumn{2}{|c|}{2,54} \\
\hline D.M.S. (período) & \multicolumn{2}{|c|}{2,20} & \multicolumn{2}{|c|}{1,88} & \multicolumn{2}{|c|}{3,40} \\
\hline
\end{tabular}

Médias seguidas de mesma letra maiúscula na coluna e minúscula na linha, não diferem estatisticamente entre si pelo teste Tukey $(\mathrm{P}<0,05)$ * - significativo ao nível de $5 \%$ de probabilidade, ** - significativo ao nível de $1 \%$ de probabilidade, ns - não significativo presença e ausência de palha de cana-de-açúcar e submetido a diferentes períodos de estiagem. Para B. decumbens foram observados excelentes resultados de controle para aplicação sem palha e sobre a palha, para os diferentes períodos estudados, exceto para o período de 60 dias sem chuva com aplicação sobre a palha. Jaremtchuk et al. (2009), avaliando a eficácia da aplicação do flumioxazin no controle de $B$. decumbens, observaram bons níveis de controle quando este foi aplicado na dose de 25 a $40 \mathrm{~g} \mathrm{ha}^{-1}$, entre 0 e 7 dias antes da semeadura desta planta daninha. 
Carbonari et al.

Para D. horizontalis foram observados, em todas as situações estudadas, níveis de controle superiores a $95 \%$, o que demonstra controle excelente desta espécie pelo flumioxazin. No entanto, os menores resultados de controle foram observados para aplicação sobre a palha e no período de 60 dias sem ocorrência de chuvas. Resultados semelhantes foram observados por Carbonari et al. (2009) para aplicação de flumioxazin sobre a palha de milho no controle de Digitaria spp. Han et al. (2002) verificaram que o flumioxazin (75 $\mathrm{g} \mathrm{ha}^{-}$ $\left.{ }^{1}\right)$ manteve controle considerável sobre $D$.

Tabela 2. Controle de I. nil e I. grandifolia pelo herbicida flumioxazin em diferentes períodos de permanência na palha de cana-de-açúcar antes da ocorrência de chuvas. Botucatu/SP 2009/10.

\begin{tabular}{|c|c|c|c|c|c|c|}
\hline \multirow[b]{2}{*}{ Períodos (dias) } & \multicolumn{2}{|c|}{$14 \mathrm{DAC}$} & \multicolumn{2}{|c|}{$28 \mathrm{DAC}$} & \multicolumn{2}{|c|}{$42 \mathrm{DAC}$} \\
\hline & $\begin{array}{l}\text { com } \\
\text { palha }\end{array}$ & $\begin{array}{c}\text { sem } \\
\text { palha }\end{array}$ & $\begin{array}{l}\text { com } \\
\text { palha }\end{array}$ & $\begin{array}{c}\text { sem } \\
\text { palha }\end{array}$ & $\begin{array}{l}\text { com } \\
\text { palha }\end{array}$ & $\begin{array}{c}\text { sem } \\
\text { palha }\end{array}$ \\
\hline & \multicolumn{6}{|c|}{ I. nil } \\
\hline 1 & $94,3 \mathrm{Ab}$ & $99,8 \mathrm{Aa}$ & $97,8 \mathrm{Aa}$ & $100,0 \mathrm{Aa}$ & $96,0 \mathrm{Aa}$ & $100,0 \mathrm{Aa}$ \\
\hline 15 & $92,3 \mathrm{ABb}$ & 98,0 Aa & $95,0 \mathrm{ABb}$ & 99,8 Aa & $96,3 \mathrm{Aa}$ & $99,5 \mathrm{Aa}$ \\
\hline 30 & $89,0 \mathrm{ABb}$ & 99,3 Aa & $92,3 \mathrm{ABb}$ & $100,0 \mathrm{Aa}$ & $92,8 \mathrm{Ab}$ & $100,0 \mathrm{Aa}$ \\
\hline 60 & $87,8 \mathrm{Bb}$ & $99,5 \mathrm{Aa}$ & $93,5 \mathrm{Bb}$ & $100,0 \mathrm{Aa}$ & $93,3 \mathrm{Ab}$ & $100,0 \mathrm{Aa}$ \\
\hline $\mathrm{F}$ (posicion.) & 71,8 & & 42 , & & 29 & $5 * *$ \\
\hline $\mathrm{F}$ (períodos) & 2,3 & & & & & $3^{\text {ns }}$ \\
\hline $\mathrm{F}$ (palha $\mathrm{x}$ per.) & 2,6 & & & & & \\
\hline C.V. (\%) & 2, & & & & & 86 \\
\hline D.M.S.(posicion.) & 4, & & & & & \\
\hline \multirow[t]{2}{*}{ D.M.S. (período) } & 5, & & & & & \\
\hline & \multicolumn{6}{|c|}{ I. grandifolia } \\
\hline 1 & $96,8 \mathrm{Aa}$ & $99,8 \mathrm{Aa}$ & $97,5 \mathrm{Aa}$ & $99,8 \mathrm{Aa}$ & $98,5 \mathrm{Aa}$ & $99,8 \mathrm{Aa}$ \\
\hline 15 & $97,5 \mathrm{Aa}$ & $99,5 \mathrm{Aa}$ & $97,3 \mathrm{Aa}$ & 99,5 Aa & $97,3 \mathrm{ABa}$ & $99,8 \mathrm{Aa}$ \\
\hline 30 & $92,8 \mathrm{Ab}$ & 98,3 Aa & $95,8 \mathrm{Aa}$ & 99,3 Aa & $97,3 \mathrm{ABa}$ & $99,0 \mathrm{Aa}$ \\
\hline 60 & $91,8 \mathrm{Ab}$ & $99,5 \mathrm{Aa}$ & $91,5 \mathrm{Ab}$ & 99,3 Aa & $91,5 \mathrm{Bb}$ & $99,0 \mathrm{Aa}$ \\
\hline $\mathrm{F}$ (posicion.) & \multicolumn{2}{|c|}{$17,78^{* *}$} & \multicolumn{2}{|c|}{$12,06^{* *}$} & \multicolumn{2}{|c|}{$9,35 * *$} \\
\hline $\mathrm{F}$ (períodos) & \multicolumn{2}{|c|}{$2,26^{\mathrm{ns}}$} & \multirow{2}{*}{\multicolumn{2}{|c|}{$1,70^{\mathrm{ns}}$}} & \multicolumn{2}{|c|}{$2,61 *$} \\
\hline $\mathrm{F}$ (palha $\mathrm{x}$ per.) & \multicolumn{2}{|c|}{$1,43^{\mathrm{ns}}$} & & & \multicolumn{2}{|c|}{$1,83^{\text {ns }}$} \\
\hline C.V. $(\%)$ & \multirow{2}{*}{\multicolumn{2}{|c|}{$\begin{array}{l}3,16 \\
4,47\end{array}$}} & \multicolumn{2}{|c|}{3.29} & \multirow{2}{*}{\multicolumn{2}{|c|}{$\begin{array}{l}3,08 \\
4,39\end{array}$}} \\
\hline D.M.S.(posicion.) & & & \multicolumn{2}{|c|}{4,70} & & \\
\hline D.M.S. (período) & \multicolumn{2}{|c|}{5,97} & \multicolumn{2}{|c|}{6,26} & \multicolumn{2}{|c|}{5,87} \\
\hline
\end{tabular}

Médias seguidas de mesma letra maiúscula na coluna e minúscula na linha, não diferem estatisticamente entre si pelo teste Tukey $(\mathrm{P}<0,05) . *$ - significativo ao nível de 5\% de probabilidade, ** - significativo ao nível de $1 \%$ de probabilidade, ns - não significativo sanguinalis. Segundo Carbonari et al. (2009), as falhas no controle, observadas nos maiores períodos sem ocorrência de chuvas, podem estar associadas à degradação do herbicida na palha, por ação de microorganismos ou fotodegradação, uma vez que o produto ficou exposto à radiação solar por um longo período na palha. Quando um herbicida é aplicado sobre a palha, é interceptado pela superfície desta e torna-se vulnerável à volatilização e/ou fotólise, até ser lixiviado para o solo (Locke \& Bryson, 1997). 
Carbonari et al.

Para I. nil e I. grandifolia (Tabela 2), de soja, nas doses de 70 e 110 g i.a. ha ${ }^{-1}$, observam-se também excelentes resultados de controle (acima de 90\%) para todas as situações estudadas. No entanto, pode-se observar tendência de redução no controle para o período de 60 dias sem chuva e com aplicação sobre a palha. Niekamp \& Jhonson (2001) obsevaram 92 e $93 \%$ de controle de Ipomoea hederacea para o flumioxazin aplicado em pré-emergência, em plantio direto

respectivamente.

Para B. pilosa e S. rhombifolia (Tabela 3) foram observados excelentes níveis de controle para todas as situações estudadas, o que demonstra uma grande sensibilidade destas espécies ao flumioxazin. Jaremtchuk et al. (2009) observaram bons níveis de controle de S. rhombifolia para aplicação do flumioxazin nas doses de $25 \mathrm{a} 40 \mathrm{~g} \mathrm{ha}^{-1}$.

Tabela 3. Controle de B. pilosa e $S$. rhombifolia pelo herbicida flumioxazin em diferentes períodos de permanência na palha de cana-de-açúcar antes da ocorrência de chuvas. Botucatu/SP - 2009/10.

\begin{tabular}{|c|c|c|c|c|c|c|}
\hline \multirow[b]{2}{*}{ Períodos (dias) } & \multicolumn{2}{|c|}{$14 \mathrm{DAC}$} & \multicolumn{2}{|c|}{$28 \mathrm{DAC}$} & \multicolumn{2}{|c|}{$42 \mathrm{DAC}$} \\
\hline & $\begin{array}{l}\text { com } \\
\text { palha }\end{array}$ & $\begin{array}{c}\text { sem } \\
\text { palha }\end{array}$ & $\begin{array}{l}\text { com } \\
\text { palha }\end{array}$ & $\begin{array}{c}\text { sem } \\
\text { palha }\end{array}$ & $\begin{array}{l}\text { com } \\
\text { palha }\end{array}$ & $\begin{array}{c}\text { sem } \\
\text { palha }\end{array}$ \\
\hline & \multicolumn{6}{|c|}{ B. pilosa } \\
\hline 1 & $97,8 \mathrm{Aa}$ & $99,8 \mathrm{Aa}$ & $97,3 \mathrm{Aa}$ & $99,3 \mathrm{Aa}$ & $98,0 \mathrm{Aa}$ & $100,0 \mathrm{Aa}$ \\
\hline 15 & $95,3 \mathrm{Ab}$ & $100,0 \mathrm{Aa}$ & $97,8 \mathrm{Aa}$ & 99,8 Aa & $98,0 \mathrm{Aa}$ & 99,8 Aa \\
\hline 30 & $95,0 \mathrm{Ab}$ & $99,8 \mathrm{Aa}$ & $98,0 \mathrm{Aa}$ & $99,5 \mathrm{Aa}$ & $98,3 \mathrm{Aa}$ & 99,5 Aa \\
\hline 60 & $95,8 \mathrm{Aa}$ & 99,3 Aa & $97,0 \mathrm{Aa}$ & 99,0 Aa & $96,0 \mathrm{Ab}$ & $100,0 \mathrm{Aa}$ \\
\hline $\begin{array}{l}\mathrm{F} \text { (posicion.) } \\
\mathrm{F} \text { (períodos) } \\
\mathrm{F} \text { (palha x per.) } \\
\text { C.V. (\%) } \\
\text { D.M.S.(posicion.) } \\
\text { D.M.S. (período) }\end{array}$ & & & $\begin{array}{r}5 \\
0 \\
0 \\
2 \\
3 \\
4\end{array}$ & & $\begin{array}{l}6, \\
0, \\
0, \\
2, \\
3, \\
4\end{array}$ & \\
\hline D.M.S. (período) & \multicolumn{6}{|c|}{ S. rhombifolia } \\
\hline 1 & 99,5 & 100,0 & 100,0 & 100,0 & $100,0 \mathrm{Aa}$ & $100,0 \mathrm{Aa}$ \\
\hline 15 & 99,3 & 100,0 & 99,0 & 99,8 & $98,3 \mathrm{Bb}$ & $100,0 \mathrm{Aa}$ \\
\hline 30 & 100,0 & 100,0 & 99,0 & 100,0 & $99,3 \mathrm{ABa}$ & $100,0 \mathrm{Aa}$ \\
\hline 60 & 96,3 & 100,0 & 98,8 & 100,0 & $99,5 \mathrm{ABa}$ & $100,0 \mathrm{Aa}$ \\
\hline $\mathrm{F}$ (posicion.) & \multicolumn{2}{|c|}{$3,82^{\mathrm{ns}}$} & \multicolumn{2}{|c|}{$2,88^{\mathrm{ns}}$} & \multicolumn{2}{|c|}{$5,83^{*}$} \\
\hline $\mathrm{F}$ (períodos) & \multicolumn{2}{|c|}{$1,75^{\mathrm{ns}}$} & \multirow{2}{*}{\multicolumn{2}{|c|}{$\begin{array}{l}0,45^{\mathrm{ns}} \\
0,37^{\mathrm{ns}}\end{array}$}} & \multicolumn{2}{|c|}{$1,41^{\mathrm{ns}}$} \\
\hline $\mathrm{F}$ (palha $\mathrm{x}$ per.) & \multicolumn{2}{|c|}{$1,75^{\mathrm{ns}}$} & \multirow{2}{*}{\multicolumn{2}{|c|}{$\begin{array}{c}0,37^{\mathrm{ns}} \\
1.26\end{array}$}} & \multicolumn{2}{|c|}{$1,41^{\mathrm{ns}}$} \\
\hline C.V. $(\%)$ & & & & \multicolumn{2}{|c|}{0,88} \\
\hline D.M.S.(posicion.) & \multirow{2}{*}{\multicolumn{2}{|c|}{$\begin{array}{l}2,63 \\
3,52\end{array}$}} & \multicolumn{2}{|c|}{1,82} & \multirow{2}{*}{\multicolumn{2}{|c|}{$\begin{array}{l}1,28 \\
1,71\end{array}$}} \\
\hline D.M.S. (período) & & & \multicolumn{2}{|c|}{2,44} & & \\
\hline
\end{tabular}

Médias seguidas de mesma letra maiúscula na coluna e minúscula na linha, não diferem estatisticamente entre si pelo teste Tukey $(\mathrm{P}<0,05)$ * - significativo ao nível de 5\% de probabilidade, ** - significativo ao nível de $1 \%$ de probabilidade, ns - não significativo

A retenção dos herbicidas pela palhada época de ocorrência de chuvas após aplicação pode ser atribuída às diferentes características dos produtos (Rodrigues, 1993; Fornarolli et físico-químicas dos produtos, às quantidades e al., 1998; Maciel \& Velini, 2005; Carbonari et origem da cobertura morta e à intensidade e al. 2009; Tofoli et al., 2009). Verifica-se que 
Carbonari et al.

apesar da baixa solubilidade do flumioxazin, $1,79 \mathrm{mg} \mathrm{L}^{-1}$ (Ferrell et al., 2005), o mesmo promoveu bons resultados de controle para aplicação sobre a palha, o que demonstra que uma parcela da dose aplicada chegou ao solo com a chuva simulada de $20 \mathrm{~mm}$, e que a quantidade do produto que chegou ao solo e a associação com a presença de cobertura morta foram bastante eficazes no controle das plantas daninhas.

\section{Conclusão}

De maneira geral, pode-se observar que a aplicação do flumioxazin sobre o solo ou sobre a palha de cana-de-açúcar promoveu bons níveis de controle das plantas daninhas. Pode-se observar também uma tendência de redução nos níveis de controle para algumas espécies de plantas daninhas, quando o flumioxazin foi exposto ao período de 60 dias sem ocorrência de chuvas, indicando que houve degradação do produto quando submetido a períodos extensos de exposição na superfície da palha ou do solo sem a ocorrência de chuva.

\section{Referências}

ALISTER, C, et al. Dissipation and movement of flumioxazin in soil at four field sites in Chile. Pest Management Science. v.64, n.5, p.579-583, 2008.

CARBONARI, C.A. et al. Efeitos de períodos de permanência do flumioxazin no solo e na palha de milho e aveia na eficácia de controle de plantas daninhas. Revista Brasileira de Herbicidas, v.8, n.3, p.85-95, 2009.

CARBONARI, C. A. et al. Efeitos de períodos de permanência de clomazone + hexazinona no solo e na palha de cana-de-açúcar antes da ocorrência de chuvas na eficácia de controle de plantas daninhas. Planta Daninha, v.28, n.1, p.197-205, 2010.
CAVENAGHI, A.L. et al. Dinâmica do herbicida amicarbazone (Dinamic) aplicado sobre palha de cana-de-açúcar (Saccarum officinarum). Planta Daninha, v.25, n.4, p.831837, 2007.

FERRELL, J.A, et al. Sorption and desorption of flumioxazin to soil, clay minerals and ionexchange resin. Pest Management Science, v.61, n.1, p.40-46, 2005.

FORNAROLLI, D. A. et al. Influência da cobertura morta no comportamento do herbicida atrazine. Planta Daninha, v.16, n.2, p.97-107, 1998.

GODOY, M.C. et al. Efeito da cobertura morta de milheto (Pennisetum americanum) sobre a eficácia do herbicida metribuzin no controle de Ipomoea grandifolia e Sida rhombifolia. Planta Daninha, v.25, n.1, p.79-86. 2007.

HAN, J. et al. Weed control in summer-sown soybeans with flumioxazin plus acetochlor and flumiclorac-pentyl plus clethodim. Weed Biology. Management, v.2, n.2, p.120-122, 2002.

JAREMTCHUK, C.C. et al. Efeito residual de flumioxazin sobre a emergência de plantas daninhas em solos de texturas distintas. Planta Daninha, v.27, n.1, p.191-196, 2009.

LAMOREAUX, R. J.; JAIN, R.; HESS, F. D. Efficacy of dimethenamid, metolachlor and encapsulated alachlor in soil covered with crop residue. Bringhton Crop Protec. Conference Weeds, v.3, n.3, p.1015-1020, 1993.

LOCKE, M. A.; BRYSON, C. T. Herbicide-soil interaction in reduced tillage and plant residue management systems. Weed Science, v.45, n.1, p.307-320, 1997.

MACIEL, C. D. G.; VELINI, E. D. Simulação do caminhamento da água da chuva e herbicidas em palhadas utilizadas em sistemas de plantio direto. Planta Daninha, v.23, n.3, p.471- 481, 2005. 
Carbonari et al.

MILLER, L. C.; RESENDE, L. C. L.; MEDEIROS, A. M. L. Manejo de herbicidas na lavoura de cana-de-açúcar. STAB, v.13, n.34, p. 9-13, 1995.

NIEKAMP, J.W., JOHNSON, W.G. Weed management with sulfentrazone and fumioxazin in no-tillage soyabean (Glycine max). Pest Management Science, v.20, n.3, p.215-220, 2001.

RODRIGUES, B.N. Influência da cobertura morta no comportamento dos herbicidas imazaquin e clomazone. Planta Daninha, v.11, n.1, p.21-8, 1993.

RODRIGUES, B. N.; ALMEIDA, F. S. Guia de herbicidas. 5.ed. Londrina: Edição dos Autores, 2005.

SIMONI, F. et al. Eficácia de imazapic e sulfentrazone sobre Cyperus rotundus em diferentes condições de chuva e palha de canade-açúcar. Planta Daninha, v.24, n.1, p.769778, 2006.

TOFOLI, G. R. et al. Dinâmica do tebuthiuron em palha de cana-de-açúcar. Planta Daninha, v.27, n.4, p.815-821, 2009.

VELINI, E.D.; NEGRISOLI, E. Controle de plantas daninhas em cana crua. In: CONGRESSO BRASILEIRO DA CIÊNCIA DAS PLANTAS DANINHAS, 22., 2000, Foz de Iguaçu. Palestras... Foz de Iguaçu: 2000. p.148-164.

WATTS D.W., HALL, J.K. Tillage and application effects on herbicide leaching and runoff. Soil \& Tillage Research, v.39, n.3-4, p.241-257, 1996. 